\title{
Symptomatic Breast Cancers and Why Breast Pain May not Always Need Clinical Review
}

\author{
Nicola Cook* (D), Jeremy Batt* (1), Clare Fowler (i) \\ Thirlestaine Breast Centre, Cheltenham General Hospital, Cheltenham, UK
}

\begin{abstract}
Objective: Breast pain contributes a heavy burden to the symptomatic breast clinic, accounting for a large number of referrals due to patient/clinician subjective anxiety and unclear aetiology. We assess the link between breast pain and cancer with a view to easing the demand on breast services.

Materials and Methods: All new breast cancer diagnoses were identified from the multidisciplinary team outcomes for the 12 months between October 2017 and October 2018. Presenting symptoms were identified from the General Practice referrals and consultant letters. Examination findings were checked with details on imaging requests.

Results: 436 new symptomatic cancer diagnoses were made in patients with a median age of 68 (range 25-97). 334 patients were referred by General Practice as two-week waits who formed the cohort selected for analysis (77\%). New lumps accounted for 294 ipsilateral cancer diagnoses (88\%), nipple symptoms for $28(8 \%)$ and pain with normal examination for 12 (4\%, all screening aged patients). All 12 cancers in the patients presenting with pain were correctly identified on mammography, including 4 cancers in the symptomatic breast and 8 Incidental cancers in the contralateral, non-symptomatic breast.

Conclusion: Pain does not appear to be frequent symptom of breast cancer presentation. It was more common for patients to have incidental, contralateral asymptomatic cancer than it was for patients with pain alone to have underlying ipsilateral cancer. In such cases, new cancers were identified accurately on mammography. Patients presenting with pain as an isolated symptom, having been carefully assessed in Primary Care, may yield little benefit in repeat clinical examination by a Breast Specialist. Direct to test with mammography could be safe, effective and efficient alternative practice.
\end{abstract}

Keywords: Breast cancer, breast clinic, breast pain, mammography, mastalgia

Cite this articles as: Cook N, Batt J, Fowler C. Symptomatic Breast Cancers and Why Breast Pain May not Always Need Clinical Review. Eur J Breast Health 2020; 16(4): 267-269.

\section{Introduction}

Breast cancer is the most common cancer in the UK and approximately 55,000 new cases are diagnosed each year (1, 2). A typical District General Hospital will see between six thousand and eight thousand new patients a year with between four hundred and five hundred of these identifying new breast cancer diagnoses (3). It is well established that breast pain, known as mastalgia or mastodynia, is a common symptom for women with a prevalence of up to $70-80 \%$ (4). It is described as cyclic or non-cyclic pain related to the mammary gland which occurs either in response to touch or spontaneously and is most common in patients aged between 30-40 years old but can be seen in women of any age (5). The exact cause of mastalgia remains unknown and due to its subjective nature and unclear aetiology is often managed poorly. In part, driven by patient anxiety or the concern of primary care in missing subclinical diagnosis, women concerned about breast pain can account for up to $50 \%$ of new outpatient referrals to the symptomatic breast clinic (6). While the incidence of breast pain being found as a symptomatic feature of breast cancer is seen in less than $3.2 \%$ of cases, referrals for breast pain still provides a significant workload for breast care centres as patients continue to be referred to secondary services for further evaluation $(6,7)$. As part of routine triple assessment the current standard is for clinicians to refer patients with painful breasts and no palpable lesion for breast imaging, although its exact value in these patients remains not well defined (8). The National Institute for Health and Care Excellence (NICE) have published specific guidelines to advise whether patients primarily presenting to their general practitioner with breast pain should be referred using a suspected cancer pathway. In all cases, the advice published within these guidelines includes the presence of abnormal clinical findings alongside pain (9). Despite this, women presenting with breast pain as an isolated symptom continue to form a significant proportion of women seen in two-week-wait secondary care centres leaving a significant mismatch between symptomatic burden and the cancer risk that breast pain poses. Across the five immediate neighbouring regional breast centres, breast pain patients are assessed in five different ways. One unit saw all referrals for breast pain in their

*Co-First Authors who have made equal contributions. 
two-week-wait clinic while others applied a vetting process to breast pain referrals. Vetting processes included deciding whether imaging should be carried out up front or whether the patient should be first assessed by a clinician to decide on further investigations and management. In such cases, patients were booked into the first available Consultant clinic, but not necessarily as a two-week wait cancer referral. One centre did not accept any referrals for isolated breast pain in patients aged less than 35 years. The aim of this study was to evaluate the link between breast pain and new cancer diagnosis in our unit to establish whether we could ease the burden that it places on breast surgery services with a view to improving the way breast pain patients are managed.

\section{Materials and Methods}

A retrospective cross-sectional study was performed which identified all new cancer diagnoses in a single breast centre over the twelvemonth period ending October 2018. Electronic records from multidisciplinary team outcomes on the national cancer registry, electronic hospital records and clinic notes and letters were reviewed. The results were entered into a database and were subsequently sub classified into screen detected or symptomatic/clinically detected diagnoses. The symptomatic patients were then further subdivided by referral method, identifying those referred via primary care (who formed the cohort for analysis) compared to those who had accessed breast services via other means which included patients found to have cancers based on CT scans performed by other hospital departments, including oncology identified recurrences. These patients were not included in the cohort. Patients referred via primary care were split into three categories based on their reported symptoms, which included new ipsilateral breast lump, nipple changes such as discharge or dysmorphia, and breast pain (defined as isolated, unilateral, persistent, non-cyclical pain in the absence of abnormal clinical examination). The breakdown of this can be seen in Figure 1. This clinical investigation was determined to not require Institutional Review Board/Ethics Committee review as data pertaining to the identity of specific individuals was not used. As such it was decided that additional retrospective consent from patients was not necessary. This study was not subjected to funding and no conflicts of interests are reported.

\section{Results}

There were 869 new cancer diagnoses over the 12-month period between October 2017 and October 2018; 433 screening detected cancers and 436 cancers that presented through the one-stop symptomatic breast clinic. The median age of patients was 68 years old (range 25-97 years). Of the 436 symptomatic cancers, 334 were referred from Pri-

\section{Key Points}

- Breast pain with no palpable pathology is a significant resource demand to symptomatic breast clinics in the UK.

- Incidence of breast cancer in a painful breast with no other symptom is rare.

- Breast cancers associated with pain are adequately detected using mammography.

- Ipsilateral breast pain is associated with cancer diagnosis in the contralateral, asymptomatic breast.

- Repeat clinical assessment in symptomatic clinic is therefore not necessary and direct to mammography is a safe, effective and appropriate alternative pathway. mary care (77\%). 102 new diagnoses (24\%) were referred from other sources which included Oncology, identification of recurrence at clinical follow up and incidental findings on CT scanning for other pathology investigated in other departments.

Of the 334 referrals to the symptomatic breast clinic from Primary care identification of a new breast lump was the primary symptom in 294 patients (88\%). 28 (8\%) patients were referred for nipple changes such as distortion or discharge. Finally, 12 patients (4\%) were referred for breast pain with a normal breast examination as reported by Primary Care referring clinician. Amongst these 12 patients referred with breast pain and normal examination, 4 patients $(1 \%)$ were diagnosed with an ipsilateral cancer (i.e. a new cancer found in the same breast that was symptomatic with pain). 8 patients, however, were found to have contralateral disease (i.e. a new cancer found in the non-symptomatic, "normal" breast). Three of the eight patients with contralateral cancers were actually found to have a lump in the non-painful breast when examined by the surgeon. All 12 cancers were accurately detected on mammography and all of these patients were of screening age and presented at an interval period between screening mammography.

\section{Discussion and Conclusion}

Breast pain is a common complaint, which creates a significant burden for breast care centres $(4,10)$. Although it is frequently stated that breast pain is not known to be a sign of cancer many patients continue to be referred to breast care centres for evaluation of breast pain (5).

Certain literature on the incidence of cancer amongst patients with symptomatic breast pain is conflicting. A small cohort study undertaken in France demonstrated a small increased risk of breast cancer associated with cyclical mastalgia but not, however, with other variations of mastalgia (11). In contrast, a larger study performed in USA found patients presenting with breast pain were less likely to be diagnosed with breast cancer than those without breast pain (12). In our cohort, ipsilateral breast pain was associated with diagnosis of contralateral cancer twice as frequently than diagnosis of ipsilateral cancer $(n=8 / 12$ vs $n=4 / 12$ respectively).

The value of breast imaging in patients with breast pain also is somewhat unclear. One study consisting of almost 1000 patients over a five-year period found only eight patients with breast pain had malignancy. Of these eight, only four were in the ipsilateral breast, similar to results demonstrated in our study (8). They went on to show that on comparison of symptomatic and non-symptomatic groups, cancer

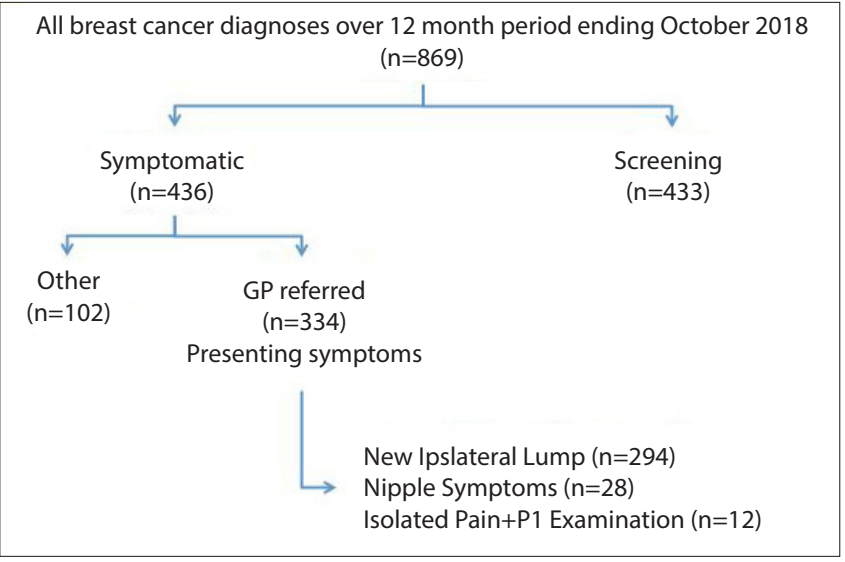

Figure 1. Patient flow diagram 
prevalence was comparable $(0.7 \%$ and $0.8 \%$ respectively) and therefore concluded that breast pain is not associated with cancer.

All 12 patients diagnosed with cancer in our study, having been referred with mastalgia and a normal clinical examination, had a correct diagnosis identified on mammographic investigation. This implies that further clinical evaluation or examination by a breast surgeon may be of little additional benefit and that stand-alone mammography (as occurs in screening) may be a sufficient diagnostic modality. It has been shown, however, that women who received imaging upfront are also more likely to undergo repeat imaging and subsequent biopsy compared with women who do not receive initial imaging (10). The obvious risk of this is the potential for over-investigation of patients and unnecessary emotional anxiety.

The American College of Radiology have published 'appropriateness' criterion with regards to imaging for breast pain. They suggest that the only situation in which imaging for breast pain is not recommended is for patients with non-focal, diffuse or cyclical pain in the absence of other suspicious clinical findings (4). However, while it is said that patients with mastalgia should not be worried regarding the possibility of cancer, it is likely that imaging will be necessary to alleviate patient concern (1). The issue regarding patients' anxiety that their mastalgia symptoms represented a malignancy and whether undergoing imaging impacted positively or negatively upon their concerns was not evaluated buy our study. It may have been interesting to investigate patients' ideas about whether they had anticipated imaging during their appointment or whether this had come as a surprise.

In conclusion, given that the average Breast Care Centre will see between 6000-8000 new symptomatic referrals per year with large studies suggesting breast pain can account for up to $50 \%$ of referrals, the assumed implication is that potentially up to 3000 patients with breast pain are being seen over a twelve-month period. In our cohort breast pain as a symptomatic feature of ipsilateral breast cancer was seen in four patients out of 334 new cancer diagnoses referred to symptomatic breast clinic from primary care (incidence $=1.2 \%$ ). On this assumption the prediction, therefore, is that up to 3500 patients with breast pain may be attending symptomatic breast clinic for repeat assessment so that we can detect 4 new cancers (incidence $=0.0003 \%$ ). When put into context and to inform work force planning in an already stretched National Health Service it must be noted that given 10 minute appointments, 3500 patients consume 35,000 minutes or 583 hours' worth of clinical time per year. When considering that none of these patients with newly diagnosed cancer in symptomatically painful breasts would have been inadequately diagnosed with mammography without clinical review we conclude that direct to mammography is a safe and time/cost efficient alternative practice. We also suggest that breast pain, portrayed as a concerning symptom associated with breast cancer, is removed from all public health literature as this may contribute to unnecessary emotional morbidity as the incidence of breast cancer presenting with pain as an isolated symptom is as low as $1 \%$.

Ethics Committee Approval: Ethics committee approval was received for this study from the ethics committee of Cheltenham General Hospital Breast Department.
Informed Consent: Informed consent was obtained from the Hospital Department on behalf of their patients' to analyze the information presented in this study.

Peer-review: Externally peer-reviewed.

Author Contributions: Concept - J.B., C.F.; Design - J.B.; Supervision C.F.; Resources - Thirlestaine Breast Centre; Materials - Thirlestaine Breast Centre; Data Collection and/or Processing - J.B., N.C.; Analysis and/or Interpretation - J.B., N.C.; Literature Search - J.B., N.C.; Writing Manuscript - J.B., N.C.; Critical Review - C.F.

Conflict of Interest: The authors have no conflicts of interest to declare.

Financial Disclosure: The authors declared that this study has received no financial support.

\section{References}

1. Altıntas Y, Bayrak M. Evaluation of 1294 Female Patients with Breast Pain: A Retrospective Study. Adv Ther 2018; 35, 1411-1419. (PMID: 30094702) [Crossref]

2. Breast Cancer Now: The research and care charity. Breast cancer symptoms and signs. [Internet] 2020 Breast Cancer Now. [updated August 2019; cited November 2019] Available from: https://breastcancernow. org/information-support/have-i-got-breast-cancer/signs-symptomsbreast-cancer

3. Royal United Hospitals Bath NHS Foundation Trust. Breast Unit. [Internet] Royal United Hospitals Bath NHS Foundation Trust 2020. [cited November 2019] Available from: https://www.ruh.nhs.uk/patients/services/breast_unit/index.asp

4. Holbrook A, Moy L, Akin EA, Baron P, Didwania AD, Heller SL, LePetross HT, Lewin AA, Lourenco AP, Mehta TS, Niell BL, Slanetz PJ, Stuckey AR, Tuscano DS, Vincoff NS, Weinstein SP, Newell MS. ACR Appropriateness Criteria Breast Pain. J Am Coll Radiol 2018; 15: 276282. (PMID: 30392596) [Crossref]

5. Kushwaha A, Shin K, Kalambo M, Legha R, Gerlach K, Kapoor M, Yang WT. Overutilization of Health Care Resources for Breast Pain. AJR Am J Roentgenol 2018; 211: 217-223. (PMID: 29792736) [Crossref]

6. Hafiz S, Barnes N, Kirwan C. The Management of idiopathic mastalgia: A systematic review. J Prim Health Care 2018; 10: 312-323. (PMID: 31039960) [Crossref]

7. Yasemin A, Mehmet B. Assessment of Breast Cancer Incidence in Patients with Mastalgia and Routine Screening. Int J Surg Res Pract 2019; 6: 094. [Crossref]

8. Duijm L, Guit G, Hendriks J, Zaat J, Mali W. Value of breast imaging in women with painful breasts: observational follow up study. BMJ 1998; 317: 1492-1495. (PMID: 9831579) [Crossref]

9. NICE: National Institute for Health and Care Excellence. Breast pain - cyclical. [Internet] NICE 2020. [updated August 2016; cited December 2019] Available from: https://cks.nice.org.uk/breast-paincyclical\#!diagnosisSub

10. Howard M, Battaglia T, Prout M, Freund K. The effect of imaging on the clinical management of breast pain. J Gen Intern Med. 2012; 27: 817824. (PMID: 22331398) [Crossref]

11. Plu-Bureau G, Le M, Sitruk-ware R, Thalabard J. Cyclical Mastalgia and Breast Cancer Risk: Results of A French Cohort Study. Cancer Epidemiol Biomarkers Prev 2006; 15: 1229-1231. (PMID: 16775187) [Crossref]

12. Khan $S$, Apkarian A. Mastalgia and breast cancer: a protective association? Cancer Detect Prev 2002; 26: 192-196. (PMID: 12269765) [Crossref] 